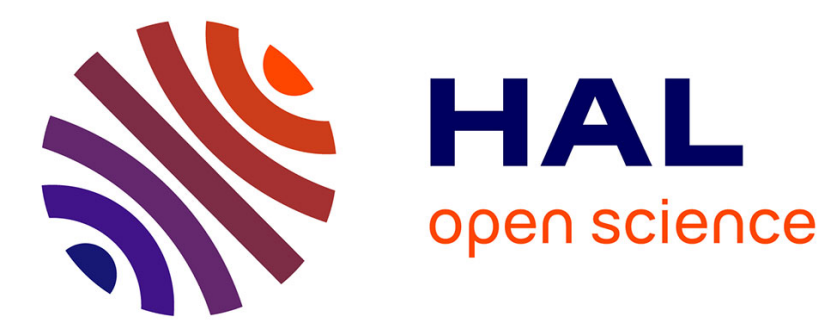

\title{
Parcours de vie des enfants et des jeunes relevant du dispositif de protection de l'enfance: les paradoxes d'une biographie sous injonction
}

Pierrine Robin, Nadège Séverac

\section{- To cite this version:}

Pierrine Robin, Nadège Séverac. Parcours de vie des enfants et des jeunes relevant du dispositif de protection de l'enfance: les paradoxes d'une biographie sous injonction. Recherches familiales, 2013, Dossier thématique du RT 22 de l'AFS, Parcours de vie et approche biographique, Catherine Negroni et Philippe Cardon, 10, pp.91-102. 10.3917/rf.010.0091 . hal-01235578

\section{HAL Id: hal-01235578 \\ https://hal.science/hal-01235578}

Submitted on 2 Mar 2016

HAL is a multi-disciplinary open access archive for the deposit and dissemination of scientific research documents, whether they are published or not. The documents may come from teaching and research institutions in France or abroad, or from public or private research centers.
L'archive ouverte pluridisciplinaire HAL, est destinée au dépôt et à la diffusion de documents scientifiques de niveau recherche, publiés ou non, émanant des établissements d'enseignement et de recherche français ou étrangers, des laboratoires publics ou privés. 


\section{Parcours de vie des enfants et des jeunes relevant du dispositif de protection de l'enfance : les paradoxes d'une biographie sous injonction}

Pierrine Robin, Maître de Conférence en sciences de l'éducation, Université Paris Est Créteil ${ }^{1}$

Nadège Séverac, Sociologue, Chargée d'étude à l'Observatoire National de l'Enfance en danger $^{2}$

Cet article vise, à travers l'expérience d'enfants et de jeunes de l'aide sociale à l'enfance, à mettre à l'épreuve l'idée de l'individualisation de l'action publique, ainsi que ce qu'elle implique en termes d'accompagnement des publics concernés. A partir de 31 récits de parcours d'enfants et de jeunes de 11 à 25 ans, nous avons cherché à comprendre les paradoxes d'une acquisition de l'autonomie sous contrainte. Il ressort des récits biographiques des enfants placés, l'expérience de conditions de socialisation extrêmement paradoxales. Alors que les enfants ne disposent pas toujours d'un espace de réflexivité pour élaborer leur propre histoire, sont peu associés aux décisions qui les concernent et voient les liens tissés durant la prise en charge peu reconnus, il leur est demandé en permanence de se raconter et de s'engager biographiquement.

Le dispositif de protection de l'enfance marque un point d'entrée de la puissance publique dans les familles où des enfants dits "en danger ou en risque de l'être » peuvent être retirés à ces dernières à des fins de protection. Il met en scène des acteurs pris dans des relations contraignantes aux institutions, captifs de décisions qu'ils ne maîtrisent pas. La domination de classe subie par les familles concernées, défavorisées, contrôlées ${ }^{3}$, stigmatisées a été analysée de longue date. Ce qui a moins été analysé, c'est la double asymétrie des positions entre des professionnels, forts de leur légitimité, et des enfants et des jeunes, en position de minorité et au final « invisibilisés » dans le processus de décision de leur propre situation ${ }^{4}$.

En miroir, les chercheurs ont longtemps témoigné de réticences à interroger les enfants et les jeunes ${ }^{5}$, notamment lorsque ceux-ci se trouvent en situation de vulnérabilité ou de maltraitance ${ }^{6}$. Les entretiens qualitatifs, ayant pour finalité la reconstitution de parcours complexes à partir du point de vue des professionnels, des parents, mais aussides enfants, commencent seulement à se développer en protection de l'enfance.Ce qui ressort principalement des récits des enfants interrogés, c'est un sentiment de dépossession de leur trajectoire de vie, une quête de sens et de cohérence dans l'élaboration de leur parcours ${ }^{7}$.

A la différence de la légitimité croissante des enfants et des jeunes de la population générale à

\footnotetext{
${ }^{1}$ Pierrine Robin, REV-CIRCEFT (EA 43 84), OUIEP, Université Paris Est Créteil, SESS Immeuble Pyramide, 80 avenue du général de Gaulle, 94009 Créteil cedex, France.Contact : pierrine.robin@u-pec.fr

${ }^{2}$ Nadège Séverac, sociologue. Contact: nadege.severac@wanadoo.fr

${ }^{3}$ Jacques DONZELOT, La police des familles, Paris, Editions de minuit, 1977.

${ }^{4}$ Sally HOLLAND, « Representing Children in Child Protection Assessments », Childhood Nr 8, 2001, pp. 322339

${ }^{5}$ Isabelle DANIC, Julie DELALANDE et Patrick RAYOU, Enquêtes auprès d'enfants et de jeunes : Objets, méthodes et terrains de recherche en sciences sociales, Rennes, PUR, 2006.

${ }^{6}$ Jude IRWIN, Fran WAUGH, Michelle BONNER, "The inclusion of children and young people in research on domestic violence", research article 2. Communities, Families and Children Australia, Nr. 1, July 2006.

${ }^{7}$ Christine ABELS-EBER, Pourquoi on nous a séparés? Récits de vie croisés : des enfants placés, des parents et des professionnels, Paris, Erès, 2006.
} 
disposer d'une marge de manœuvre pour élaborer leur trajectoire et construire leur identité ${ }^{8}$, les enfants et les jeunes de la protection de l'enfance expérimentent des parcours de vie particulièrement contraints, dans un contexte qui voit pourtantsetransformer l'action sociale. I. Astier 'évoque ainsi un «grand retournement de la dette sociale », l'usager étant, en contrepartie de l'aide, enjointà l'autonomieet qui plus est appelé à en faire la preuve en puisant dans sa propre trajectoire biographique, constituée en un récit livré à l'institution ${ }^{10}$. Du côté des professionnels, mobiliser ainsi les usagers suppose un changement de posture dans la relation d'aide, le travail d'accompagnementévoluant d'un travail sur autrui à untravail avec autrui.

Dans cet article, nous souhaiterions interroger la déclinaison de ce nouveau paradigme de l'action publique dans le champ de l'aide sociale à l'enfance :les textes de loi témoignent depuis les années 1980 d'une inflexion visant à rendre les enfants et les famillespartie prenante de lamesure de protection. La loi du 5 mars 2007 poursuit en ce sens avec le principe d'une aide administrative contractuelle primant sur l'intervention judiciaire et fait du projet pour l'enfant le support de cette contractualisation. Mais curieusement l'enfant mineur est absent de la signature de son projet qui est simplement " porté à sa connaissance » (art. 223-1 CASF). Ainsi, alors que l'enfant est l'usager le plus directement concerné par une forme de prise en charge parmi les plus lourdes qui soient, le texte prévoit que le contrat puisse légitimement se construire et être signé entre l'institution et les parents, tandis quele jeune, quel que soit son âge, est mis devant le fait accompli.

C'est donc avec un double questionnement que nous abordons cet article, à la fois méthodologique et analytique. D'un point de vue épistémologique et méthodologique, nous chercherons à savoir dans quelle mesure l'approche biographique permet une compréhension renouvelée des ambiguïtés de l'intervention sociale et des rapports de force en protection de l'enfance. D'un point de vue empirique et analytique, nous chercherons à comprendre ce que les parcours des enfants en protection de l'enfance donnent à voir de leurs contraintes, mais aussi de leurs marges d'autonomie et de leurs stratégies identitaires et d'affiliations.

Deux recherches empiriques ${ }^{1112}$ seront mobilisées, ayant en commun d'avoir sollicité des jeunes pour mener avec eux des entretiens biographiques : 31 récits de parcours de jeunes de 11 à 25 ans, anciennement ou encore pris en charge par les services de l'aide sociale à l'enfance de la Drôme, et de la Seine Saint-Denis constituent le corpus exploité ici. Le choix de l'approche biographique renvoie à son double caractère heuristique et éthique, consistant à prendre en compte l'enfant dans l'ensemble de ses sphères de vie ${ }^{13}$ au lieu de le réduire à son statut d'usager des services sociaux ${ }^{14}$. Il s'agit donc de donner la parole aux jeunes en les laissant libres de leur présentation de soi, ce qui doit leur permettre de récupérer une épaisseur de sujet, matrice d'une intelligibilité propre.Ainsi l'analyse ne se limite pas à la perception des difficultés et des aides reçues, mais tient compte des ressources et des capacités

\footnotetext{
${ }^{8}$ François de SINGLY, Les adonaissants, Paris, Armand Colin, 2006

${ }^{9}$ Isabelle ASTIER, Les nouvelles règles du social, Paris, PUF, 2007.

${ }^{10}$ Isabelle ASTIER, "Du récit privé au récit civil : la construction d'une nouvelle dignité ?", Lien social et politique, RIAC n ${ }^{\circ} 34,1995$.

${ }^{11}$ Pierrine ROBIN, L'évaluation de la maltraitance en tension, Thèse de doctorat en sciences de l'éducation, soutenue le 20 mars 2009 à Nanterre

${ }^{12}$ Pierre MOISSET, Nadège SEVERAC, Etude sociologique sur les parcours de jeunes en grandes difficultés au sein de l'aide sociale à l'enfance, « Une socialisation impossible ? Cinq parcours de jeunes en grandes difficultés », 2010.

${ }^{13}$ Daniel BERTAUX, Les récits de vie, Paris, Nathan, collection 128, 1997

${ }^{14}$ Hans GUNTHER; Hans HOMFELDT; Wolfgang SCHROER; Cornelia SCHWEPPE (Hg.), Vom Adressaten zum Akteur, Soziale Arbeit und Agency. Opladen, 2008.
} 
d'initiative et de transformation des acteurs.

Trois dimensions du parcours des jeunes seront observés,dimensions identifiées par $\mathrm{M}$. Duval ${ }^{15}$ ou encore M. Stein ${ }^{16}$ comme supportsde la construction identitaire et de l'autonomie, soit : le rapport à l'origine du placement et à l'histoire, les possibilités de participer aux décisions durant l'évaluation de leur situation et la création de liens durant le placement.

\section{Rapport à l'histoire : savoir d'où l'on vient pour savoir où l'on est}

Une des premières dimensions essentielles dans le parcours des enfants confiés est le rapport à l'histoire et au motif du placement.

\section{Age et conditions du placement : des conditions modulant la maîtrise de sa biographie}

On note tout d'abord chez les enfants, adolescents et jeunes rencontrés un rapport à leur propre histoire et aux raisons initiales du placement assez contrasté : certains en donnent une présentation très parcellaire et morcelée, d'autres sont parvenus à élaborer une présentation très construite et conceptualisée.

Ainsi, les enfants placés très tôt, et qui ont eu peu accès à leur histoire familiale,la présentent de manière assez lacunaire: "Tout ce que je sais, c'est que je suis née dans un camion de pompier. Je m'appelle Aude car mon frère a voulu m'appeler Aude, mon père lui il aurait voulu m'appeler Deborah. J'ai été placée car mes parents étaient alcooliques. " (Aude, 21 ans, en contrat jeune majeur). Dans ce récit, on peut percevoir la non-maîtrise par cette jeune fille de son histoire familiale et du placement, qui fait étrangement écho à la non-maitrise par ses parents de sa naissance, comme de son prénom. De même, July, 14 ans, placée vers 3 ans,actuellement en établissement collectif, dit n'avoir " aucune idée » de pourquoi elle a été placée. Relancée pour savoir si elle a questionné les professionnels, elle répond : "Ben ils m'ont dit... de toute façon, il faut que j'attende dix-huit ans pour consulter mon dossier où il y a pas tout, mais quand même quelques éléments ».

On voit ici confirmé un constat effectué de longue date, à savoir l'absence de représentation précise des raisons du placement, ce qui a été en bonne partie renvoyé à la réticence des professionnels à communiquer à l'enfant le motif du placement ${ }^{17}$ et à l'interroger sur sa vision de sa situation et du danger.Ce rejet massif s'explique d'une part par une vision de l'enfant comme incapable de comprendre la situation, et d'autre part par la crainte des travailleurs sociaux et des cadres de l'Aide sociale de l'enfance de reproduire un traumaauprès d'enfants déjà vulnérabilisés ${ }^{18}$.

Ce vide ou ce flou autour des origines du placement s'avère lourd d'implications en termes de construction identitaire. Pour les jeunes placés précocement qui n'ont pas ou peu le souvenir de la vie et surtout de la relation avec leurs parents, c'est le moment même du tissage du lien

\footnotetext{
${ }^{15}$ Michelle DUVAL, «L'action collective pensée par Hannah Arendt : comprendre l'agir ensemble pour le favoriser », Service social, Volume 54, numéro 1, 2008, p. 83-96 http://id.erudit.org/iderudit/018345a

${ }^{16}$ Mike STEIN, Resilience and young people leaving care, Overcoming the odds, York, Joseph Rowntree Foundation, 2005.

${ }^{17}$ Emilie POTIN, Enfants en Danger. Enfants protégés. Enfants sécurisés ? Parcours de (dé)placement(s) des enfants confiés à l'Aide sociale à l'enfance, Thèse de doctorat en sociologie, Brest, Université de Bretagne occidentale, 2009.

${ }^{18}$ Jan MASON et Anne MICHAUX, The Starting Out with Scarba project : Facilitating children's participation in child protection process, Paddington : The Benevolent Society, 2005.
} 
et du désir d'enfant de leurs parents à leur égard qui échappe, soit l'origine et la raison d'être de leur existence. L'énigme qui en résulte ne peut être que douloureuse, dans la mesure où les rapports de filiation sont des rapports de reconnaissance fondamentaux ${ }^{19}$ où se jouent une identification réciproque qui, pour l'enfant, est au principe de sa construction de soi, mais aussi du monde ${ }^{20}$. Or dans ce cas de figure, les liens sont marqués par le sceau de la rupture, de telle sorte que la reconnaissance n'est plus ni évidente, ni parfois possible, laissant ces jeunes intimement $«$ désaffiliés $»^{21}$.

Pour d'autres, c'est le regard public porté sur leur relation avec les parents et le pourquoi de sa qualification en termes de « risque », de « danger » qui n'est pas intelligible. Le rapport au motif de placement est ainsi différencié selon que l'enfant se soit perçu d'emblée comme « en danger »- comme Assia (16 ans, placée en établissement) qui a remarqué très tôt un dysfonctionnement dans sa famille : "En maternelle, j'ai remarqué que les parents étaient affectueux. (...)Je voyais bien la différence avec les autres. Ça frappait aux yeux »- ou que le qualificatif de danger soit l'objet d'une intériorisation au fil du parcours comme pour Océane (19 ans, sortie du dispositif) : "Dans mes souvenirs lointains, les gens de l'ASE, c'étaient des méchants (...) En grandissant, j'ai compris que c'était pour mon bien. (...) Plus tard, j'ai discuté avec ma grande sœur que mon père était dans un sale état, que c'était dangereux. Cela a été une déduction plus tard. Un enfant qui vit chez des étrangers, je ne trouvais pas ça normal. (...) Ça me semblait illogique dans ma tête. (...) Ça m'a fait avancer dans la vie. Je ne regrette pas, ce que je regrette c'est qu'on n'ait pas pris le temps de m'expliquer. (...) on finit par comprendre mais tard. ».Ce qui est en jeu ici évoque ce qu'a pu montrer $\mathrm{M}$. Blueband-Langner ${ }^{22}$ aux Etats-Unis, dans un travail réalisé auprès d'enfants en phase terminale dans un service de cancérologie. Ces enfants partageaient avec ceux de la protection de l'enfance l'expérience d'une réticence des adultes à verbaliser sur le diagnostic de leur situation. L'acquisition d'informations sur leur propre histoire est dès lors un processus long qui inclut à la fois l'expérience de la prise en charge et des changements dans la conception de soi.

\section{Un passé qui ne passe pas}

Clairement, cette question des raisons du placement, dans la mesure où elle oblige à qualifier les relations parents/enfants,ne s'épuise pas au fil du temps, mais se ramifie dans le présent et s'actualise au cours des évolutions de la prise en charge.Les jeunes sont en effet condamnés à une quête de leur histoire et de leurs liensauprès de leurs multiples instances de socialisation raison pour laquelle elle a d'ailleurs peu de chances d'aboutir :le jeune est confrontéà une pluralité de versions,la plupart du temps incompatibles entre elles. C'est ce qu'expliqueKarima (16 ans, placement levé récemment) : Les parents, ils nous la font quand on rentre chez nous : "Ouais les éducateurs c' est des méchants lalala... », et quand on arrive chez la famille d'accueil... " oui c'est mieux qu'elle reste avec nous parce que les parents, ils sont comme ci, comme ça, lala ». Donc on a deux versions comme ça et nous on est au milieu franchement... (...) Quand ils se réunissent c'est mieux. (...) avec mon éduc., Mme B, ça c'est arrêté (...)il y avait des fois où avec ma mère, ils étaient pas d'accord!... mais ils trouvaient un arrangement ». Chacun des protagonistes engagé auprès de Karimaa un point de vue

\footnotetext{
${ }^{19}$ Axel HONNETH, La lutte pour la reconnaissance, Paris, Cerf, 2000.

${ }^{20}$ Dans le rapport avec les « autres significatifs », qui sont ceux qui ont pour tâche de socialiser l'enfant, se joue en effet l'installation de l'individu à l'intérieur du monde de la société, et du même coup, l'appropriation de son identité par l'enfant. Peter BERGER \& Thomas LUCKMANN, La construction sociale de la réalité, Paris, Méridiens Klincksieck, 1986.

${ }^{21}$ Robert CASTEL, Les métamorphoses de la question sociale, Paris, Fayard, 1995.

${ }^{22}$ Myra BLUEBAND-LANGER, The Private Worlds of Dying Children, Princenton University Press, 1980.
} 
différent et une définition de l'autre, souvent négative. Elle est la seule à faire le lien entre tous ces acteurs qui ne se parlent pas, et elle se retrouve ainsiisolée dans une position de go between $^{23}$.Elle ne peut ainsi avoir affaire qu'à une réalité clivée, d'ordre conflictuel, non seulement dans le présent des relations entre les différents professionnels de l'Aide Sociale à l'Enfance (ASE) et sa famille, mais aussi en elle,ce qui,dit-elle,lui " fait mal ».Les conséquences en sont une diffraction identitaire quasi inévitable obligeantlesjeunesà un travail d'unification particulièrement intense, qui peut-être aussi usant et trop lourd, comme l'exprime Karima : "Tout ce qu'on a dans nos têtes, on n'a pas 45 ans pourtant».

Ce halo négatif qui entoure à la fois les origines de la séparation et le temps du placement est destinéà persister tant qu'un travail de parole, avec une personne significative n'est pas effectué. En effet, les jeunesqui parviennent à avoir une représentation construite de leur propre histoire sont ceux qui ont pu trouver un espace de réflexivité durant le placement -ce qui nécessite un lien de confiance intense avec un «autrui significatif » - et un espace de dialogue entre les différents acteurs.

Maiscompte tenu de la manière dont est organisé le placement, ce lien avec un autrui significatif ne peut fréquemmentpas se construire. Pour en revenir au témoignage de July, elle n'a pas pu trouver du côté des professionnels quelqu'un qui puisse lui en dire plus sur son histoire, mais surtout un autrui suffisamment engagé auprès d'elle pour l'accompagner dans sa quête.Elle ne peut donc que renvoyer à son dossier, même si là encore le parcours est semé d'embûches. Les mineurs protégés par l'aide sociale à l'enfance doivent en effet attendre,s'ils ne sont pas accompagnés d'un adulte, leur majorité pour accéder à la mémoire consignée de leur histoire, mémoire que l'on sait par ailleurs incomplète ${ }^{24}$. Durant leur minorité, ils n'ontaccès qu'aux " morceaux choisis » pour eux par l'institution : "Je ne pouvais pas tout voir. C'est la juge qui me faisait des comptes-rendus. J'ai eu que quelques photocopies. Je ne comprends pas qu'ils ne me laissent pas lire tranquillement. (...) Il y a toujours plein de zones d'ombre. Ma famille d'accueil ne connaissait pas toutes les réponses, ma famille biologique évitait d'y répondre. Je savais vaguement. ( ...) Le juge, je lui ai demandé de lire pour voir ce qui s'est passé de 6 mois à 14 ans. » (Aude, 21 ans, en contrat jeune majeur)

Dans le discours des jeunes, la prise en charge à ceci de paradoxal que,sans leur donner la possibilité de construire une vision claire de leur passé, elle les y ramène sans cesse, ce qui est vécu comme une violence et comme une entrave à la construction de leur situation comme susceptible d'être ouverte sur un avenir différent du passé ${ }^{25}$ : "A partir de 16 ans, j'ai commencé à évacuer un peu mieux, carrément tout, mais il y a toujours ce placement qui rabâche les choses. (...) Le jour où j'ai eu ma majorité, j'ai dit :"C'est fini ma vie de merde, maintenant, je vais me construire. 》(Fred, 19 ans, sorti du dispositif)

Lorsque I. Astier évoque les modalités du travail avec les bénéficiaires de l'action sociale comme « une rencontre» qui «pour un nombre sans cesse croissant d'agents, (...) ne consiste plus à obtenir le consentement des sujets de leur intervention à des valeurs générales mais à les accompagner dans la construction de leur identité personnelle ${ }^{26} »$, force est de

\footnotetext{
${ }^{23}$ Cléopâtre MONTANDON, « La sociologie de l'enfance anglophone », Education et sociétés 2, 1998, pp. 91118

${ }^{24}$ Jean-Yves BARREYRE, Patricia FIACRE, Vincent JOSEPH, Yara MAKDESSI, Une souffrance maltraitée, parcours et situation de vie de jeunes dits « incasables », Rapport de recherche pour l'ONED, 2008.
}

\footnotetext{
${ }^{26}$ Isabelle ASTIER, Les transformations de la relation d'aide dans l'intervention sociale, Informations sociales $2009 / 2-\mathrm{n}^{\circ} 152$, pp. 52 à 58, p. 53
} 
constater que nombre de jeunes de l'ASE n'ont pas le sentiment d'avoir fait ce type de rencontre. Les jeunes sont donc amenés à construire leur identité souvent sans le support de l'institution, on pourrait même dire malgré elle.

\section{Participation : une faible association aux décisions en cours de prise en charge ?}

Un second élément qui marque fortement les parcours des enfants confiés est la possibilité ou non de participer aux décisions en cours de prise en charge.

\section{Avoir l'initiative de l'entrée dans le dispositif : une clé pour la maîtrise de son parcours}

Au regard de nos études empiriques, il apparaît que le mode d'entrée dans le dispositif de protection de l'enfance influe fortement sur laperception par les enfants des possibilités ultérieures d'action. Ainsi comme l'amontré J. Münder ${ }^{27}$ en Allemagne, il convient de distinguer les enfants qui se présentent comme les déclencheurs de l'aide de ceux pour qui l'aide a été perçue comme subie.

Les enfants qui disent avoir pris d'eux-mêmes l'initiative de recourir aux services sociaux ont l'impression par la suite de garder la maîtrise de leurs parcours tout au long de la prise en charge: "J'ai déclenché mon arrivée ici (...) De ma situation, c'est tout moi qu'ai décidé du début à la fin.» (Assia, 16 ans, accueillie en établissement) Ils se perçoivent comme acteur du processus d'évaluation de leur propre situation et ont pu s'approprier les aides ultérieures reçues, qu'ils ont l'impression d'avoir choisies.

Inversement les enfants, adolescents et jeunes s'étant perçus initialement comme objets de l'aide, se sont sentis exclus de l'évaluation de leur situation et les aides reçues ont été dans un premier temps perçues comme subies, comme pour Abdel (13 ans, accueilli en établissement)«En premier, c'était parti pour une famille d'accueil. La famille d'accueil allait me récupérer. J'ai rien dit, la décision était déjà prise. (...) Ils en ont parlé pendant que je n'étais pas là. (...) J'étais petit, je trouvais pas les bons mots, ça s'est fait vite. Je ne voulais pas y aller. Ils m'ont dit que j'étais obligé. " Pour ces enfants, accepter l'aide proposée, nécessite un long travail de réappropriation qui peut permettre,au fildu parcours en protection de l'enfance, de se poser en acteur de l'évaluation en cours de mesure; Abdel a fini par «reprendre la main »: "Avant ça se passait pas avec moi, maintenant ça se passe plus avec moi. Pendant la première audience, je n'ai jamais parlé, je pleurais (...). Mais pendant la troisième audience c'est ma mère qu'a pleuré et c'est moi qu'ai parlé. »

\section{Des jeunes considérés comme des infans : rapports de force et stratégies}

Il reste que depuis le moment de l'entrée jusqu'à la préparation de la sortie de placement, les enfants ne sont guère associés aux décisions concernant leur situation, quand bien même ils sont concernés au premier chef. Dans cette configuration difficile, certains d'entre eux finissent par se résigner et se taire : «Au début, j'aurai voulu me faire entendre plus. Mais les gens ont du mal à croire» (Aurélie, 19 ans, en contrat jeune majeur). Car ce n'est pas seulement la position dominante des professionnels qui met en difficulté les enfants, adolescents et jeunes, mais également la position d'alliance entre adultes. Ces jeunes ont l'impression que les professionnels se positionnent du côté de leurs parents, qu'ils croient en priorité leur récit: "Les enfants ne sont pas écoutés simplement parce qu'ils sont des enfants. $C$ 'est comme si je mentais et qu'ils croyaient seulement mes parents. Je ne comprends pas ça. Un enfant aussi sait ce qu'il fait. » (Océane, 19 ans, sortie du dispositif)

\footnotetext{
${ }^{27}$ Johanes MUENDER et B. MUTKE, Kindeswohl zwischen Jugendhilfe und Justiz- Ergebnisse eines Forschungsprojektes, Sozialpädagogisches Institut im SOS-Kinderdorf, 2001
} 
Certains,compte tenu de l'importance des enjeux de ce qui se décide pour leur vie présente et leur vie future, développent différentes stratégies pour conquérir un droit à la parole, malgré tout. Cela peut consister à construire une stratégie d'alliance avec un parent ou la fratrie, afin de peser davantage et de faire valoir une force de négociation :" Avec mes sæurs on avait toujours le même discours. (...) Ça a été plus facile de négocier car ils nous écoutaient vraiment. Ils sentaient que ce n'était pas un caprice d'enfant. On donnait chacun notre opinion, c'était assez ressemblant. »(Claire, 20 ans, en contrat jeune majeur). La stratégie peut aussi consister à agir de manièreà infléchir les décisions, par des actes amenant àaménager les décisions non souhaitées : "Au début je voulais pas être placée et séparée de mon frère,donc je me suis créé des visites dans la famille d'accueil de mon frère " (Aude 19 ans, en contrat jeune majeur). On peut parler dans ce cas de "socialisation interprétative verticale inversée $»^{28}$ dans la mesure où les enfants parviennent à modifier les rapports de force et participent en ce sens à la modification de l'ordre établi.

\section{Le "passage à l'acte ", dernière forme d'affirmation en l'absence de parole}

Mais pour d'autres, face à la non prise en compte de leur point de vue, il ne reste que la solution désignée par les professionnels comme «passage à l'acte», sous la forme de fugue, pour infléchir les décisions prises et refuser l'ordre établi : "J'étais en foyer à $T$. dans un autre département. (...) Je ne voulais pas du tout revenir. Ils m'ont ramenée ici en voiture. Je suis retournée le lendemain en train à T. Ils ont été souples. Ils ont refait une tentative. Ils ont essayé de me garder. Ils ont écouté ce que je demandais. " (Ariane, 21 ans, sortie du dispositif). On pourrait rapprocher ces situations d'un $«$ vote par les pieds $»^{29}$ :le choix que le mineur usager n’a pas dans la participation négociée, il le prend par ses mouvements.

La violencesuit la même logique, c'est-à-dire une tentative d'être pris en compte, puis en cas d'échec, une manière d'affirmer un refus de la soumission : "Parce qu'en fait, quand je leur parle, je n'ai pas l'impression qu'ils comprennent. Mais quand je leur dis : " je vais faire ça" là ils réagissent. (...)Les autres c'est : "C'est moi l'adulte! Tu fais! ». Ben je dis : "Toi tu fais, mais c'est pas avec moi, donc ça sert à rien de parler avec moi, si tu fais pas avec moi ", donc je casse, je détruis..." (Karima, 16 ans, mainlevée de placement).Du côté des professionnels, si la signification de ces mises acte est entendue comme refus, elle ne débouche guère sur une écoute, tant ces manœuvre de résistances sont perçues avant tout sous l'angle du désordre, voire du chaos. La stigmatisation qui s'ensuit peut avoir pour effet de faire entrer certains jeunes, parmi les plus marqués par des ruptures multiples, dans une « carrière » de violence ${ }^{30}$. A contrario, donner la parole à ces jeunes que l'institution désigne comme « ingérables » dévoile l'existence d'une sorte d'individualisation négative au sens où le jeune devient le porteur exclusif de l'absence de dialogue, d'une inutilité de sa parole - non qu'il soit réduit au silence, puisque Karima peut parler, mais sans que cela change quoi que ce soit à des décisions qui lui échappent ${ }^{31}$. Le coût de cette politique de la terre brûlée s'avère élevé pour les professionnels, constamment appelés à gérer les innombrables incidents et confrontés au sentiment d'échec de leur travail, et plus encore pour les jeunes qui se voient régulièrement exclus des lieux de prise en charge, éventuellement mis sous camisole chimique, ou encore judiciairement sanctionnés.

\footnotetext{
${ }^{28}$ William A. CORSARO, The Sociology of Childhood. Thousand Oaks-California : Pine Forge Press, 1997

${ }^{29}$ Expression utilisée à l'origine dans les ex-républiques soviétiques pour qualifier les mouvements des populations face à une absence de démocratie politique.

${ }^{30}$ Howard BECKER, Outsiders. Etudes de sociologie de la déviance, Paris, Métailié, 1985

${ }^{31}$ Le constat est identique s'agissant des parents des enfants protégés. Cf. Régis SECHER, Reconnaissance sociale et dignité des parents d'enfants placés, Parentalité, précarité et protection de l'enfance, Paris, L'Harmattan, 2010.
} 
L'obligation de respecter un ensemble de règles institutionnelles, par définition impersonnelles, ne favorise pas chez les jeunes de la protection de l'enfance le jeu décrit par E. Ramos ${ }^{32}$ s'agissant des jeunes en population générale : ces derniers, en avançant en âge se créent dans la relation avec leurs parents une marge de négociation et d'expérimentation leur permettant de construire progressivement leur propre rapport à la règle. Les jeunes de l'ASE sont donc clairement placés dans une situation paradoxale :disposant de moins de soutien et de moins de tempsque les jeunes de population générale pour élaborer leur cheminement vers l'autonomie, compte tenu de l'effet couperet de la fin de prise en charge qui tombe de plus en plus à 18 ans $^{33}$, ils disposent néanmoins de moins de possibilité d'exercer concrètement cette autonomie durant leur prise en charge. L'accompagnement tel que les jeunes le perçoivent, relevant davantage d'un travail sur plutôt qu'avec : "Le travail sur autrui consiste avant tout à attribuer un rôle à autrui. Le professionnel est en position de surplomb et impose sa définition de la situation. Même si les logiques de service et relationnelle sont présentes, elles sont comme dominées par la logique de contrôle social. Avant même que la relation ne s'installe, l'identité institutionnelle de l'usager est donnée. Cet usager est, d'une certaine manière, prédéfini dans le travail sur autrui. Il doit demeurer passif. Au mieux, il accepte et adhère à ce qu'on lui impose, au pire, il se soumet $\rangle^{34}$.

\section{Les attaches créées : des affiliations essentielles mais peu sécurisées}

Une troisième dimension du parcours fondamentale dans la construction de l'identité est la possibilité ou non de construire des liens d'affiliation.

\section{Les liens créés au cours du placement : une " parenté additionnelle "}

Au fil de la mesure de placement, a fortiori lorsque celle-ci est intervenue précocement et s'étend dans le temps, les enfants et jeunes vivent au quotidien avec des personnes en position de devenir leurs « autrui significatifs »: «Avec la famille d'accueil, on s'appelle, on se rend visite. On ne coupe pas les ponts avec des gens qui nous éduquent. (...) Ils ont un peu comme un rôle de parents mais sans remplacer les parents. Ils sont là pour aimer les enfants. (...)S'il n'y a pas d'affect, l'enfant ne peut pas évoluer. C'est comme s'il était éduqué par des robots. »(Océane, 19 ans, sortie du dispositif). La jeune fille souligne l'importance de ce qui se joue dans la possibilité de cette affiliation, qui relève en quelque sorte d'une reconnaissance de son humanité, à travers celle de ses sentiments, sans quoi les relations entre enfants placés et éducateurs se désubjectivent complètement, se« robotisent ».Cependant, comme elle le précise,en écho avec le concept de suppléance parentale ${ }^{35}$, les liens créés au cours et en raison du parcours ne viennent pas se substituer à ceux existant avec la famille d'origine, mais s'y ajouter; la famille d'accueil c'est « un peu comme les parents » mais « sans les remplacer".

Comme Océane, les enfants et jeunes rencontrés insistent sur la nécessité de tenir compte de ces liens tissés durant l'accueil, dont ils expriment la valeur en recourant à ce qui a été désigné comme «parenté plurielle ${ }^{36}$.Cette parenté additionnelle se donne à entendre dans le

\footnotetext{
${ }^{32}$ Elsa RAMOS, Rester enfant, devenir adulte, La cohabitation des étudiants chez leurs parents, Paris, L'Harmattan, 2002

${ }^{33}$ Les restrictions budgétaires raréfient en effet les contrats jeunes majeurs permettant une prolongation des prises en charge jusqu'à 21 ans.

${ }^{34}$ Isabelle ASTIER, op. cit. p. 53

${ }^{35}$ Paul DURNING, Education familiale, acteurs processus enjeux, Paris, L’Harmattan, 2006.

${ }^{36}$ Anne CADORET, Parenté plurielle. Anthropologie du placement familial, Paris, L’Harmattan, 1995.
} 
récit des jeunes, lorsqu'ils utilisent tour à tour les termes mes «vrais parents » pour désigner les parents géniteurs, vrai étant employé au sens légal et génétique et "vrais parents » pour désigner la famille d'accueil, vrai renvoyant alors au lien subjectif et affectif. Le caractère identique des termes utilisés ne renvoie pas pour autant à une confusion dans leur esprit du rôle qu'ils attribuent à chacun, la distinction qu'ils opèrent entre "les parents géniteurs » et " ceux qui apprennent à faire toutes les démarches dans la vie " étant très nette. Cette confusion apparente est liée plutôt à la difficulté d'expliquer et de mettre en mots ces liens subjectifs créés du fait d'une absence de termes reconnus et partagés dans la pratique pour désigner ces parentalités additionnelles, comme peut l'exprimer ce jeune en situation d'entretien : "C'est un peu dur à expliquer, bon attendez, je vais prendre une feuille, ce sera plus facile. " (Antoine, 19 ans, en contrat jeune majeur). On peut penser que cette absence de termes communs pour désigner la parentalité additionnelle renvoie à " une zone aveugle » de l'évaluation et de la prise en charge. De fait, le point de vue de l'enfant est invisibilisé, alors que celui-ci, isolé en position de go between entre les différents acteurs, est le seul à pouvoir faire le lien entre ses liens.

\section{Le déni des attaches nouées}

Les récits des jeunes montrent que les attaches nouées au cours de leur parcours ne sont guère prises en compte. Les pratiques en vigueur à cet égard ne sont pas différentes de celles que l'on a vues à l'œuvre en matière de décisions. Sur cette question particulière joue cependant, en sus d'habitudes de travail qui ne conçoivent pas le jeune pris en charge comme un sujet doté d'un point de vue et d'une parole sur son devenir, la philosophie du dispositif de protection de l'enfance français qui vise à travailler le lien de l'enfant avec sa famille d'origine $^{37}$, la finalité visée étant le retour en famille. Depuis 2007, la réforme de la loi prévoit pourtant que « les liens d'attachement noués par l'enfant (...) soient maintenus, voire développés dans son intérêt supérieur » (art. 3), mais il n'en demeure pas moins qu'en pratique, les enfants n'ont aucune garantie à cet égard: "Il s'est trouvé que j'avais un éducateur qui prenait fait et cause pour mon père biologique, ce qui n'était pas le meilleur choix à faire. Il a fait toutes les démarches en fonction de ce que disait mon père et la famille d'accueil passait après, enfin quand elle passait! "(Antoine, 19 ans, en contrat jeune majeur). Il s'ensuit que la préservation de ces liens précieux repose sur les jeunes, dont l'intérêt est d'adopter un langage conforme aux représentations des professionnels, ou même de taire ce qu'ils ont pu construire avec leur "parenté additionnelle », quand bien même elle peut leur paraître plus «vraie» que l'autre. Exprimer la réalité de ces liens affectifs les expose sinon au risque de les voir jugé excessifs par les professionnels et de se voir déplacés : "Un éducateur m'a dit que j'allais changer de famille d'accueil. Ça s'est fait en petites réunions. La décision s'est imposée à moi. Je ne voulais pas partir. Je me suis sentie comme une valise qu'on transporte d'un endroit à l'autre. On ne m'a pas demandé mon avis. On m'a présenté ces gens. On m'a emmené chez eux. On m'a dit tu vas y aller. On a changé avec mon frère car le service considérait qu'ils maternaient trop mon frère. Il ne fallait pas montrer de sentiments. Il fallait juste laver le linge. "(Océane, 19 ans, sortie du dispositif). La question des attaches est certainement de toutes, celle qui autorise le moins les stratégies de diverses natures : le seul langage à avoir est celui socialement audible dans le contexte de l'institution ; en ce sens, les jeunes ne peuvent que participer à la reproduction de l'ordre établi.

Toutefois, sur cette question en particulier, l'institution, à trop traiter les jeunes comme des "valises » (selon l'expression d'Océane), court le risque que ceux-ci en viennent,pour reprendre une expression couramment utilisée par les professionnels, à « ne plus se poser nulle

\footnotetext{
${ }^{37}$ Cf . Définition des objectifs de la protection de l'enfance dans la loi du 5 mars 2007, notamment art. 1.
} 
part ». Privés d'attaches, de liens donnant du sens à un quotidien partagé, certains d'entre eux entrent alors dans un engrenage de violences et de déplacements : (Ken, 18 ans, mainlevée de placement, SDF) : "Et donc, M. ça dure 4 mois, après en famille d'accueil, 2 jours. Après toutes les familles d'accueil, ça dure pas, après Mme B. (famille d'accueil), deux ans. Après je suis parti chez Mme M. (famille d'accueil), ça a duré 8 mois. Et après je suis retourné chez Mme B. Et j'ai fait encore une année entière. Et après je suis retourné en foyer à M. Je suis resté 2 ans. Après je suis parti chez Mme G. (famille d'accueil), je suis resté 3 ans. Et après je suis parti au foyer de R., où je suis resté 4 ans, et après je suis parti au foyer des G. où je suis resté deux ans. Et après vous êtes là, aujourd'hui, et il n'y a plus rien ».

Non sans ironie, les jeunes qui se prennent au jeu de l'institution, et qui ne s'attachent plus, en viennent à être ceux qui posent le plus de problèmes. L'analogie d'Océane qui parlait de « robot» pour désigner la relation éducative " intermédiaire » proposée par l'institution - ni attachement, ni déliaison - sous la forme d'un quotidien partagé sans affects, parait très pertinente. C'est précisément cette forme d'objectivation, de déshumanisation que certains jeunes ne peuvent supporter et à laquelle ils réagissent par un désengagement relationnel et une course vers la destruction. Comme l'exprime Karima, ne tenir à rien, revient à ne plus être tenu, ni retenu par rien: "Parce qu'à un moment, j'en avais rien à cirer, enfin elle, j'en avais rien à cirer tandis que Mme B., elle est là 24 heures sur 24, le matin, le soir, elle est toujours là donc en tous les cas si j'ai un problème (...) 》.

\section{Conclusion}

Il ressort des récits biographiques des enfants placés,l'expériencede parcours contraints etdes conditions de socialisation extrêmement paradoxales. Déplacés de chez eux pour des motifs rarement explicités, avec des conséquences à long terme sur leurs possibilités d'affiliations ultérieures, leur avenir se décidele plus souventsans eux. Qui plus est, l'institution ne reconnaît guèrela nécessité absolue d'une affiliation des enfants et des jeunes avec des « autres significatifs ». Alors que ce qui est en jeu est non seulement l'existence d'un espace de réflexivité permettant de revenir sur une histoire malmenée, mais plus fondamentalement, à travers la socialisation, un ancrage au monde et la définition de soi à l'intérieur de ce monde. A ce titre, le type d'accompagnement dont les enfants protégésbénéficient relève bien d'un travail sur et non avec eux.Cela n'empêche pas l'institution d'attendre d'eux une autonomie pleine et entière à 18 ans, au plus tard 21 ans, c'est-à-dire bien plus précocement que pour les jeunes n'ayant pas eu à être protégés, sans qu'ils n'aient pour ainsi dire jamais l'occasion de l'exercer durant leur parcours de prise en charge.

Entendre les jeunes permet en même temps d'échapper à la tentation de conclure à la toute puissance de l'institution en faisant apparaître que même au sein de ce cadre extraordinairement contraint, les jeunes mettent en place des stratégies pour avoir un droit à la parole et tenter de reprendre la mainsur leur parcours de vie. L'instaurationd'un espace de dialogue s'obtient cependant au prix de maints efforts et reste ponctuelle, toujours à reconquérir. La difficulté des adultes à reconnaître les diverses formes d'expression des jeunes comme telles témoigne bien du rapport de force subi par ces jeunes, dépendants de l'interprétation des adultes et des suites qu'ils resteront seuls à décider. Pour les jeunes de l'Aide sociale à l'enfance, avoir une part créative dans sa propre vie demeure synonyme de risque, matériel et symbolique, car le fait de se démarquer, de se voir reconnaître comme sujet peut toujours faire l'objet d'une stigmatisation et se retourner contre soi.

Ultime paradoxe, alors que l'institution tend à faire silence sur l'histoire des jeunes, à leur imposer un parcours et à ignorer leurs affiliations, elle n'en demande pas moins aux jeunes de 
se livrer, «d'en dire quelquechose », bref de s'engager biographiquement. Les jeunes rencontrés se sont d'ailleurs fréquemment montrés très lassés, voire mis à mal par cette injonction à se raconter durant la prise en charge. On peut se demander pourquoi alors ils ont signifiéapprécierde raconter leur parcours au chercheur. La différence de ces deux contextes, celuide la relation d'aide et celui de la recherche est indéniable : on est d'un côté face à un récit public, centré sur les failles, demandé en contre-partie de l'aide ; de l'autre face à un récit privé, centré sur les difficultés mais autant sur les ressources etdontla logique se situe dans le registre du don,contre-don, plutôt que de l'exigence. En effetsi l'entretien biographique a suscité l'intérêt des jeunes,c'est qu'il leur a permis d'être restauré dans une position de sujet, sollicités par le chercheur pour dire le sens a posteriori d'événements discontinus et subis. Ainsi comme a pu l'analyser G. Truc ${ }^{38}$ à partir des travaux de P. Ricoeur,l'acteur qui a besoin de donner sens à son existence n'a pour seul recours que l'interprétation au temps présent du passé. La narration permet à l'individu qui n'est pas l'auteur de ses actions et dont il ne maitrise pas les conséquences, d'en rester le coauteur par le sens ${ }^{39}$. Le récit peut permettre alors à ces jeunes qui ont eu à subir de se voir imposer ouvertement des pans entiers de leur vie, de se positionner au moment de l'énonciation comme les narrateurs de leur propre histoire, dont ils sont seuls à conserver la mise en sens. Un autre aspect nous semble fondamental : alors que le récit institutionnel n'a de cesse que de renvoyer les jeunes à leur passé, le récit biographique ouvre sur un caractère prospectif par la promesse d'avenir qu'il contient. La " promesse de l'aube », pour paraphraser R. Gary, « mise en intrigue » dans le récit, stabilise la pluralité des expériences qui éclatent l'identité. Toutefois, on peut se demander avec G. Truc si tous les acteurs, du fait des conditions dans lesquelles ils sont placés, sont en mesure de tenir, ou même de se fairedes promesses.

\section{Plan :}

\section{Introduction}

1) Rapport à l'histoire : savoir d'où l'on vient pour savoir où l'on est.

a) Age et conditions du placement : des conditions modulant la maîtrise de sa biographie

b) Un passé qui ne passe pas

2) Participation : une parole au jeune sur des décisions décisives?

a) Avoir l'initiative de l'entrée dans le dispositif : une clé pour la maîtrise de son parcours

b) Des jeunes considérés comme des infans : rapports de force et stratégies

c) Le "passage à l'acte ", dernière forme d'affirmation en l'absence de parole

d) Accès à la majorité : une autonomie sous injonction

3) Les attaches créées : des affiliations essentielles mais peu sécurisées.

a) Les liens créés au cours du placement : une «parenté additionnelle»

b) Le déni des attaches nouées

\footnotetext{
${ }^{38}$ Gérôme TRUC, 2005, Une désillusion narrative ? De Bourdieu à Ricœur en sociologie, tracés 2005-8, p. 47-67

${ }^{39}$ Paul RICOEUR, Soi même comme un autre, Paris, Seuil, 1990
} 


\section{Conclusion}

